\title{
Dinâmica da comunidade e populações arbóreas da borda e interior de um remanescente florestal na Serra da Mantiqueira, Minas Gerais, em um intervalo de cinco anos (1999-2004)
}

\author{
ARY T. OLIVEIRA FILHO ${ }^{1,4}$, WARLEY A.C. CARVALHO ${ }^{2}$, EVANDRO L.M. MACHADO $^{1}$, \\ PEDRO HIGUCHI ${ }^{1}$, VIVETTE APPOLINÁRIO ${ }^{1}$, GISLENE C. CASTRO ${ }^{1}$, ANA C. SILVA ${ }^{1}$, \\ RUBENS M. SANTOS ${ }^{1}$, LUÍS F. BORGES ${ }^{1}$, BRUNO S. CORREA ${ }^{1}$ e JAQUELINE M. ALVES ${ }^{3}$
}

(recebido: 2 de junho de 2005; aceito: 15 de fevereiro de 2007)

\begin{abstract}
Tree population and community dynamics in the edge and interior sectors of a forest remnant in the Mantiqueira Range, SE Brazil, over a five-year interval (1999-2004)). The dynamics of the tree community and 26 component populations was investigated in a fragment of tropical semideciduous forest in Piedade do Rio Grande, SE Brazil, based on surveys done in 1999 and 2004 in $30400 \mathrm{~m}^{2}$ plots, 12 of which were located on the edge of the fragment and 18 in its interior. The purpose was to assess whether the tree community and populations (a) were stable in the period, and (b) showed a more accelerated dynamics on the fragment edge than in its interior. Rates of mortality and recruitment of trees and gain and loss of basal area were obtained for the whole sample, its two sectors, diameter classes and tree populations. The stability hypotheses was rejected because, in both the edge and interior, mortality rates surpassed recruitment rates, gain rates of basal area surpassed loss rates, and size distributions changed, with declining density of smaller trees. These overall changes were possibly related to (a) ongoing long-lasting fragmentation effects, (b) a particular phase of a rhythmic forest cycle and, or (c) the alleged recent global acceleration of forest turnover due to increased $\mathrm{CO}_{2}$ in the atmosphere. The two sectors did differ in their turnover rates higher on the edge than in the forest interior, probably because of more abundant light at the edge, but also because tree populations of fast-growing pioneer and light-demanding species are more abundant at the edge.
\end{abstract}

Key words - forest dynamics, forest fragmentation, tree community dynamics, tree population dynamics, tropical semideciduous forest

RESUMO - (Dinâmica da comunidade e populações arbóreas da borda e interior de um remanescente florestal na Serra da Mantiqueira, Minas Gerais, em um intervalo de cinco anos (1999-2004)). A dinâmica da comunidade arbórea e de 26 populações componentes foi investigada em um fragmento de floresta estacional semidecídua em Piedade do Rio Grande, Minas Gerais, com base em inventários conduzidos em 1999 e 2004 em 30 parcelas de $400 \mathrm{~m}^{2}, 12$ das quais foram locadas na borda do fragmento e $18 \mathrm{em}$ seu interior. O objetivo foi verificar se a comunidade e populações arbóreas (a) estavam estáveis no período, e (b) mostraram uma dinâmica mais acelerada na borda do fragmento que em seu interior. Foram obtidas taxas de mortalidade e recrutamento de árvores e taxas de ganho e perda de área basal para a amostra total, seus dois setores, classes de diâmetro e populações. A hipótese da estabilidade foi rejeitada porque, tanto na borda como no interior, as taxas de mortalidade superaram as de recrutamento, as taxas de ganho superaram as de perda de área basal e as distribuições de tamanho mudaram devido ao declínio na densidade de árvores menores. Tais mudanças gerais se relacionaram, possivelmente, (a) a efeitos de longa duração da fragmentação ainda em curso, (b) a uma fase particular de um ciclo florestal rítmico e/ou (c) à suposta aceleração global das taxas de rotatividade florestal devido ao aumento do $\mathrm{CO}_{2}$ atmosférico. Os dois setores de fato diferiram nas taxas de rotatividade mais elevadas na borda que no interior da floresta, provavelmente devido à maior abundância de luz na borda, mas também porque populações de espécies pioneiras e exigentes de luz, de rápido crescimento, são mais abundantes na borda.

Palavras chave - dinâmica de comunidades arbóreas, dinâmica florestal, dinâmica de populações arbóreas, floresta tropical semidecídua, fragmentação florestal

\section{Introdução}

A acelerada fragmentação das florestas tropicais é a uma das maiores ameaças atuais à biodiversidade.

1. Universidade Federal de Lavras, Departamento de Ciências Florestais, 37200-000 Lavras, MG, Brasil.

2. Universidade Federal de Minas Gerais, Departamento de Botânica, ICB, 30161-970 Belo Horizonte, MG, Brasil.

3. Universidade Federal de Lavras, Departamento de Educação, 37200-000 Lavras, MG, Brasil.

4. Autor para correspondência: ary@ufla.br
Vários fatores advindos da fragmentação, tais como os efeitos de borda, impedimento ou redução na taxa de migração entre fragmentos, diminuição do tamanho populacional efetivo com conseqüente perda de variabilidade genética e invasão de espécies exóticas, contribuem para a deterioração de uma paisagem composta por fragmentos florestais (Lovejoy et al. 1986, Bierregaard Jr. et al. 1992, Turner 1996, Young et al. 1996, Laurance 1997, Nason et al. 1997, Brasil 2002). 
A dinâmica de comunidades florestais fragmentadas é fortemente influenciada pelo efeito borda, o que acaba levando à alteração da composição de espécies das áreas sob influência das bordas (Laurance \& Yensen 1991, Laurance et al. 1998a, 1998b). Entre as alterações ambientais com efeitos mais óbvios sobre a comunidade arbórea está o aumento da luminosidade nas bordas e proximidades (Murcia 1995). No entanto, há evidências de que o impacto mais forte entre os causadores do incremento da mortalidade, e da mortalidade diferencial, tanto das árvores da borda como das do interior do fragmento, sensível até $>300 \mathrm{~m}$ da borda, é a redução da disponibilidade de água devido ao incremento da evapotranspiração e à redução do estoque de água no solo (Kapos 1989, Kapos et al. 1997, Bierregaard Jr. et al. 2001). Estas alterações resultam na aceleração da dinâmica das áreas sob influência das bordas e na alteração da estrutura e composição de espécies nestas áreas dando origem a uma comunidade diferenciada em relação à original e ao interior dos fragmentos (Murcia 1995). A alteração tanto das condições ambientais como da composição de espécies muitas vezes se reflete em uma dinâmica fortemente diferenciada entre borda e interior dos fragmentos, mesmo depois de muitas décadas após a fragmentação (Oliveira Filho et al. 1997). Desta maneira, apenas a área nuclear de grandes fragmentos florestais seria algo representativa da floresta primitiva e as perdas em relação à área original seriam bem maiores do que sugere a área atual dos fragmentos.

O Estado de Minas Gerais tem cerca de 50\% do seu território localizado no Domínio da Mata Atlântica, incluindo aí fisionomias de Floresta Ombrófila Densa, Ombrófila Aberta, Ombrófila Mista, Estacional Decidual e Estacional Semidecidual, as quais ocupavam 4.314.235 ha em 1995, evoluindo para 4.193.174 ha em 2000, implicando em uma perda de 2,8\% somente neste curto período (Brasil 2002, Fundação SOS Mata Atlântica 2002). A região do Alto Rio Grande, no centrosul de Minas Gerais, não foge a esta realidade e sua cobertura vegetal encontra-se hoje reduzida a fragmentos de florestas, cerrado e campos, a maior parte dos quais mostra sinais de distúrbios por fogo, pecuária ou retirada seletiva de madeira para fins múltiplos (Oliveira Filho et al. 1994).

O presente estudo foi conduzido em um fragmento de floresta semidecídua em Piedade do Rio Grande formado há cerca de 150 anos e cuja comunidade arbórea já se diferenciou fortemente entre borda e interior, tanto em fisionomia como em composição de espécies (Carvalho et al., dados não publicados). A hipótese principal do estudo foi de que a dinâmica da comunidade arbórea deste fragmento florestal no período de cinco anos, entre 1999 e 2004, diferiu entre borda e interior. Em detalhe, as hipóteses de trabalho foram: (a) houve equilíbrio, no período, entre mortalidade e recrutamento de indivíduos e entre perdas e ganhos de área basal na comunidade e nas populações; (b) a dinâmica foi mais rápida na comunidade e populações da borda que nas do interior do fragmento florestal; e (c) a dinâmica de populações de espécies preferenciais de borda foi mais rápida que a de espécies preferenciais de interior.

\section{Material e métodos}

Caracterização da área de estudo - $\mathrm{O}$ objeto do presente estudo é uma área remanescente de floresta nativa com aproximadamente 30 ha localizada no município de Piedade do Rio Grande, Minas Gerais, nas coordenadas de 21 $29^{\circ} 03^{\prime \prime} \mathrm{S}$

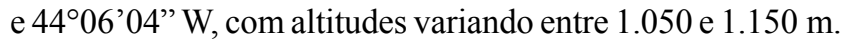
O fragmento florestal é conhecido localmente por Mata dos Pinheiros e pertence a duas propriedades rurais, as fazendas Mato Virgem e Bocaina. O clima é do tipo Cwb segundo a classificação de Köppen (mesotérmico com verões brandos e estiagem de inverno). As médias anuais de precipitação e temperatura situam-se próximas a $1.510 \mathrm{~mm}$ e $17,91{ }^{\circ} \mathrm{C}$, respectivamente, valores estes registrados para a cidade de Barbacena (Brasil 1992), que fica a cerca de $50 \mathrm{~km}$ da área de estudos em uma altitude semelhante $(1.126 \mathrm{~m})$. O embasamento rochoso é o gneiss granítico e os solos incluem Latossolos Vermelho-Amarelos Ácricos típicos, Argissolos Vermelho-Amarelos Distróficos típicos e Argissolos VermelhoAmarelos Eutróficos típicos. A vegetação pode ser classificada como floresta semidecídua alto-montana a baixomontana, segundo o sistema de Oliveira Filho \& Fontes (2000), ou então como floresta estacional semidecidual montana, na classificação do IBGE (Veloso et al. 1991). Podese considerar que a floresta encontra-se em fase madura, respeitado o mosaico de sucessão natural, pois, segundo levantamento histórico, não houve corte raso nem retirada seletiva de madeira nos últimos 150 anos, durante os quais a Mata dos Pinheiros permaneceu como propriedade da mesma família.

Inventários da comunidade arbórea - Em 1999, Carvalho et al . (dados não publicados) utilizaram um procedimento amostral estratificado e aleatório para realizar o primeiro inventário da comunidade arbórea da Mata dos Pinheiros. A área amostral foi de 1,2 ha, constituída de 30 parcelas de $400 \mathrm{~m}^{2}$ distribuídas aleatoriamente por sorteio sobre ortofotocarta 1:10.000, sendo alocadas 18 parcelas de $20 \times 20 \mathrm{~m}$ no interior do fragmento (estrato Interior) e 12 parcelas de $10^{\prime} 40 \mathrm{~m}$ nas bordas (estrato Borda). A localização das parcelas do Interior foi sorteada entre as coordenadas de uma grade de pontos de $20 \times 20 \mathrm{~m}$ ajustada ao acaso à ortofotocarta. A situação das parcelas de 
Borda foi sorteada ao longo do perímetro da borda do fragmento e seu lado maior foi justaposto à borda de forma a maximizar a representação deste ambiente. Todos os indivíduos com DAP (diâmetro à altura do peito ou a $1,30 \mathrm{~m}$ de altura) $\geq 5 \mathrm{~cm}$ foram marcados com etiquetas de alumínio (fixadas $10 \mathrm{~cm}$ acima do nível de medição do DAP) e medidos em circunferência e altura (caules múltiplos medidos quando raiz da soma dos quadrados dos diâmetros $\geq 5 \mathrm{~cm}$ ). Não foram incluídos indivíduos mortos e lianas. As medições foram realizadas entre maio e setembro de 1999. Os autores também realizaram um levantamento topográfico detalhado e analisaram amostras do solo superficial de cada parcela. Os resultados deste inventário são fornecidos por Carvalho et al. (dados não publicados).

Em julho de 2004 foi realizado o segundo inventário nas mesmas 30 parcelas alocadas em 1999. Os indivíduos mortos foram somente contabilizados, os sobreviventes foram medidos novamente e os recrutas (indivíduos que alcançaram o DAP mínimo) foram marcados e medidos. O intervalo de tempo entre os pontos centrais da duração dos dois inventários foi de cinco anos.

Dinâmica da comunidade arbórea - Variáveis de dinâmica foram calculadas para a amostra total da comunidade e para extrações da amostra total definidas pelas parcelas amostrais, estratos amostrais (Borda e Interior), classes de diâmetro e populações selecionadas. As variáveis expressam a dinâmica de duas formas: em demografia (contagens) de indivíduos arbóreos e em área basal das árvores, assumida como a soma das áreas seccionais à altura do peito calculada assumindo uma relação circular com o DAP. Com base em Sheil et al. $(1995,2000)$, foram assumidas mudanças em tamanho populacional por intervalo de tempo em proporção constante do tamanho inicial da população e foram, assim, calculadas as taxas anuais médias de mortalidade $(M)$ e recrutamento $(R)$ de árvores individuais e taxas anuais médias de perda $(P)$ e ganho $(G)$ de área basal das árvores por meio das expressões exponenciais:

$$
\begin{aligned}
& M=\left\{1-\left[\left(\mathrm{N}_{0}-m\right) / N_{0}\right]^{1 / t}\right\} \times 100, \\
& R=\left[1-\left(1-r / N_{\mathrm{t}}\right)^{1 / t}\right] \times 100, \\
& P=\left\{1-\left[\left(A B_{0}-A B_{\mathrm{m}}+A B_{\mathrm{d}}\right) / A B_{0}\right]^{1 / \mathrm{t}}\right\} \times 100, \mathrm{e} \\
& G=\left\{1-\left[1-\left(A B_{\mathrm{r}}+A B_{\mathrm{g}}\right) / A B_{\mathrm{t}}\right]^{1 / t}\right\} \times 100,
\end{aligned}
$$

onde $t$ é o tempo decorrido entre os inventários, $N_{0}$ e $N_{\mathrm{t}}$ são, respectivamente, as contagens inicial e final de árvores individuais, $m$ e $r$ são, respectivamente, o número de árvores mortas e recrutas, $A B_{0}$ e $A B_{\mathrm{t}}$ são, respectivamente, as áreas basais inicial e final das árvores, $A B_{\mathrm{m}}$ é a área basal das árvores mortas, $A B_{\mathrm{r}}$ é a área basal dos recrutas e $A B_{\mathrm{d}}$ e $A B_{\mathrm{g}}$ são, respectivamente, o decremento (por meio de quebra ou perda parcial do tronco) e o incremento em área basal das árvores sobreviventes. Para expressar a dinâmica global, foram obtidas as taxas de rotatividade (turnover) em número de árvores $\left(T_{\mathrm{N}}\right)$ e área basal $\left(T_{\mathrm{AB}}\right)$ a partir, respectivamente, das médias das taxas de mortalidade e recrutamento e de perda e ganho (Oliveira Filho et al. 1997, Werneck \&
Franceschinelli 2004):

$$
\begin{aligned}
& T_{\mathrm{N}}=(M+R) / 2 \\
& T_{\mathrm{AB}}=(P+G) / 2
\end{aligned}
$$

Conforme Korning \& Balslev (1994), também foram ainda obtidas as taxas de mudança líquida no período, tanto para número de árvores $\left(C h_{\mathrm{N}}\right)$ como área basal $\left(C h_{\mathrm{AB}}\right)$, sendo utilizadas as equações:

$$
\begin{aligned}
& C h_{\mathrm{N}}=\left[\left(N_{\mathrm{t}} / N_{0}\right)^{1 / \mathrm{t}}-1\right] \times 100, \mathrm{e} \\
& C h_{\mathrm{AB}}=\left[\left(A B_{\mathrm{t}} / A B_{0}\right)^{1 / \mathrm{t}}-1\right] \times 100 .
\end{aligned}
$$

Para analisar as variações nas taxas de dinâmica entre as parcelas amostrais foram realizadas análises de variância e de correlação em caráter exploratório considerando as duas principais variáveis identificadas por Carvalho et al. (dados não publicados) como mais fortemente relacionadas com a distribuição de espécies no fragmento: a dicotomia bordainterior e o gradiente catenário dos solos. Como não emergiram padrões significativos vinculados à variação dos solos, as análises se restringiram à dicotomia borda-interior. Foi realizada uma comparação multivariada entre os estratos Borda e Interior por meio do teste de $\mathrm{T}^{2}$ de Hotelling (Morrison 1976) aplicado ao conjunto das oito taxas de dinâmica nas 30 parcelas. As variáveis foram também comparadas individualmente entre os dois estratos pelo teste de $t$ para duas amostras independentes, assumindo-se deliberadamente populações normais com variâncias iguais, com base na robustez do teste (Zar 1996). A independência entre as freqüências de indivíduos sobreviventes, mortos e recrutas nos dois estratos foi verificada pelo teste de qui-quadrado e a diferença entre os números de recrutas e mortos foi verificada por comparações entre contagens de Poisson (Zar 1996) para os dois estratos e para a amostra total. A probabilidade de erro tipo I (nível de significância) adotada em todos os testes estatísticos foi de $P \geq 0,1$.

A dinâmica das árvores por classe de diâmetro nos dois estratos foi analisada empregando-se os mesmos intervalos de classe com amplitudes crescentes $(5-10,>10-20,>20-40$ e $>40-80 \mathrm{~cm}$ ) que foram adotados em estudo de dinâmica florestal na região por Appolinário et al. (2005) para compensar o forte decréscimo da densidade nas classes de tamanhos maiores, típico da distribuição em exponencial negativo, conhecida como J-invertido. Para descrever as variações temporais ocorridas em cada classe de diâmetro, foi contabilizado o número de árvores que passou pelos seguintes eventos: permanência na classe, morte, recrutamento, imigração (ingrowth) e emigração (outgrowth) na classe, podendo os dois últimos ser progressivos ou regressivos (Lieberman et al. 1985). Com base na distribuição de diâmetros em 1999, foram utilizados testes de qui-quadrado para verificar se as frequiências de árvores vivas em 2004 e de árvores mortas foram independentes das classes de diâmetro. A diferença entre o número de árvores ingressantes (recrutas + imigrantes) e árvores egressas (mortos + emigrantes) em cada classe de diâmetro foi verificada por comparações entre contagens de Poisson para os dois estratos e para a amostra total. 
Dinâmica das populações arbóreas - Foram escolhidas para análise da dinâmica populacional na amostra total as 26 espécies que apresentaram 20 ou mais indivíduos nos dois inventários, incluindo mortos e recrutas. Para análise da dinâmica populacional nos dois estratos amostrais, foram escolhidas as cinco espécies que apresentaram 20 ou mais indivíduos tanto na Borda como no Interior. Os mesmos parâmetros de dinâmica foram calculados para as populações na amostra total e nos dois estratos amostrais. A diferença entre os números de recrutas e mortos em cada uma das 26 populações foi verificada por comparações entre contagens de Poisson. A homogeneidade das proporções de parcelas com aumento ou redução da área basal das mesmas populações foi verificada por testes de qui-quadrado. A independência entre as proporções entre árvores mortas e sobreviventes nos dois estratos, e entre recrutas e sobreviventes nos dois estratos foi verificada pelo teste de qui-quadrado para as cinco populações escolhidas.

Com o propósito de auxiliar a identificação de padrões ecologicamente significativos, as 26 espécies foram classificadas segundo sua preferência pelos habitats de interior e/ou borda e sua guilda de regeneração. Os habitats preferenciais foram definidos com auxílio do teste de MannWhitney (Zar 1996) aplicado às abundâncias das espécies nas parcelas de Borda e Interior. As guildas de regeneração, adaptadas de Lieberman \& Lieberman (1987), foram: (a) 'subbosque', (b) 'sub-dossel', (c) 'dossel-tolerante à sombra', (d) 'dossel-exigente de luz', e (e) 'pioneira'. A classificação das 12 espécies nas categorias acima se apoiou na experiência de campo dos autores. A rotatividade em número de árvores e área basal e a mudança líquida em número de árvores e área basal de cada espécie foram representadas graficamente.

\section{Resultados}

Dinâmica da comunidade arbórea - As variáveis de dinâmica da comunidade arbórea na amostra total e nos seus dois estratos, Borda e Interior, encontram-se na tabela 1. De uma forma geral, os resultados sugerem uma redução líquida da densidade e aumento líquido da área basal em ambos os estratos, como resultado de taxas de mortalidade superiores às de recrutamento e taxas de ganho superiores às de perda de área basal. De fato, as contagens de mortos foram significativamente maiores que as de recrutas na amostra total $(Z=3,180, P<0,02)$ e no Interior $(Z=3,359$, $P<0,01)$, mas não na Borda $(Z=1,457, P>0,1)$. Contudo, a distribuição dessa tendência foi espacialmente heterogênea, pois apenas metade das parcelas da Borda (seis) e de Interior (nove) registrou ao mesmo tempo redução do número de árvores e aumento da área basal. O oposto, isto é, aumento da densidade e redução da área basal, ocorreu em uma parcela de Borda e duas de Interior; duas parcelas de Borda e cinco de Interior reduziram ambas as quantias ao passo que três de Borda e duas de Interior aumentaram ambas as quantias. A distribuição dos quatro possíveis eventos nas parcelas diferiu significativamente da homogeneidade $\left(\chi^{2}=11,07\right.$, $P=0,0113)$ e a freqüência dos quatro eventos nas parcelas não diferiu significativamente entre Borda e Interior $\left(\chi^{2}=1,270, P=0,99\right)$, caracterizando uma fase não estável nos dois estratos entre 1999 e 2004, com predominância da densidade decrescente e área basal crescente.

A dinâmica expressa pelas oito taxas diferiu entre as 12 parcelas da Borda e 18 parcelas do Interior, de acordo com o teste multivariado de Hotelling $\left(T^{2}=38,07, P=0,00915\right)$. Entretanto, as comparações individuais identificaram diferenças significativas para quatro taxas: rotatividade de número de árvores $(t=1,78$, $P=0,094)$, perda de área basal $(t=1,76, P=0,098)$, ganho de área basal $(t=2,94, P=0,007)$ e rotatividade de área basal $(t=2,94, P=0,006)$, todas maiores nas parcelas de Borda que nas de Interior (tabela 1). Não foram encontradas diferenças significativas entre os dois estratos para as taxas de mortalidade $(t=0,97$, $P=0,341)$, recrutamento $(t=1,36, P=0,185)$, mudança líquida de árvores $(t=0,58, P=0,57)$ e mudança líquida de área basal $(t=1,24, P=0,225)$. Quando as árvores amostradas nos dois estratos são tratadas em conjunto, outras diferenças aparecem (tabela 1). As freqüências de sobreviventes, mortos e recrutas diferiram significativamente entre Borda e Interior $\left(\chi^{2}=14,733\right.$, $P=0,011)$. Na relação entre recrutas e sobreviventes estes foram mais freqüentes que o esperado na Borda e menos freqüentes que o esperado no Interior $\left(\chi^{2}=11,962, P=0,0005\right)$. Contudo, não houve diferenças significativas entre Borda e Interior para as freqüências de mortos em relação aos sobreviventes $\left(\chi^{2}=2,348, P=0,125\right)$. Desta maneira, dos dois estratos se diferenciam principalmente pela dinâmica mais acelerada na Borda que no Interior, particularmente na dinâmica expressa em área basal. No entanto, as mudanças resultantes do processo dinâmico não diferem entre os estratos.

As distribuições de diâmetro diferem entre os dois estratos amostrais na maior densidade na Borda que no Interior de árvores das duas menores classes de DAP, sobretudo da de $5<$ DAP $<10 \mathrm{~cm}$ (figura 1). Entre 1999 e 2004 houve uma redução na densidade de árvores da classe de $5<$ DAP $<10 \mathrm{~cm}$ e aumento da densidade de árvores da classe de $20 \leq \mathrm{DAP}<40 \mathrm{~cm}$ tanto na Borda como no Interior, mas na classe de 
Tabela 1. Dinâmica da comunidade arbórea em um fragmento de floresta semidecídua inventariada nos anos de 1999 e 2004 em Piedade do Rio Grande, MG, contabilizada para a amostra total e seus dois estratos, Borda e Interior, e expressa em número de árvores e área basal. Valores entre parênteses representam as freqüências esperadas de árvores sobreviventes, mortas e recrutadas baseados nas freqüências totais. Valores entre colchetes são as médias das $N$ parcelas de cada estrato amostral.

Table 1. Tree community dynamics in a fragment of tropical semideciduous forest surveyed in 1999 and 2004 in Piedade do Rio Grande, SE Brazil, given for the total sample and its two strata, Edge and Interior, and expressed as number of trees and tree basal area. Values within parentheses are expected frequencies for surviving, dead and recruit trees based on the total frequencies. Values within brackets are means of the $N$ plots of each sample stratum.

\begin{tabular}{|c|c|c|c|c|c|}
\hline \multirow{2}{*}{ Amostragem: } & \multirow{2}{*}{ Amostra Total } & \multicolumn{2}{|c|}{ Estrato Borda } & \multicolumn{2}{|c|}{ Estrato Interior } \\
\hline & & & & & \\
\hline Número de parcelas $(N)$ & 30 & 12 & & 18 & \\
\hline \multicolumn{6}{|l|}{ Número de árvores: } \\
\hline Inicial(1999) & 1780 & 976 & & 804 & \\
\hline Final (2004) & 1720 & 954 & & 766 & \\
\hline Sobreviventes & 1572 & 851 & $(879,8)$ & 721 & $(692,2)$ \\
\hline Mortas & 208 & 125 & $(116,4)$ & 83 & $(91,6)$ \\
\hline Recrutas & 148 & 103 & $(82,8)$ & 45 & $(65,2)$ \\
\hline Taxa de mortalidade $\left(\%\right.$ ano $\left.^{-1}\right)$ & 2,5 & 2,7 & {$[2,6]$} & 2,2 & {$[2,3]$} \\
\hline Taxa de recrutamento $\left(\%\right.$ ano $\left.^{-1}\right)$ & 1,8 & 2,3 & {$[2,2]$} & 1,2 & {$[1,3]$} \\
\hline Taxa de rotatividade $\left(\%\right.$ ano $\left.^{-1}\right)$ & 2,1 & 2,5 & {$[2,4]$} & 1,7 & {$[1,8]$} \\
\hline Taxa de mudança $\left(\%\right.$ ano $\left.^{-1}\right)$ & $-0,7$ & $-0,5$ & {$[-0,5]$} & $-1,0$ & {$[-0,9]$} \\
\hline \multicolumn{6}{|l|}{ Área basal: } \\
\hline Inicial(1999) & 50,00 & 17,89 & & 32,11 & \\
\hline Final (2004) & 51,05 & 18,66 & & 32,39 & \\
\hline $\operatorname{Mortas}\left(\mathrm{m}^{2}\right)$ & 3,21 & 1,69 & & 1,52 & \\
\hline Decremento dos sobreviventes $\left(\mathrm{m}^{2}\right)$ & 0,46 & 0,14 & & 0,32 & \\
\hline Recrutas $\left(\mathrm{m}^{2}\right)$ & 0,44 & 0,31 & & 0,13 & \\
\hline Incremento dos sobreviventes $\left(\mathrm{m}^{2}\right)$ & 4,28 & 2,29 & & 1,99 & \\
\hline Taxa de perdas $\left(\%\right.$ ano $\left.^{-1}\right)$ & 1,5 & 2,1 & {$[2,2]$} & 1,2 & {$[1,4]$} \\
\hline Taxa de ganhos $\left(\%\right.$ ano $\left.^{-1}\right)$ & 1,8 & 2,8 & {$[3,1]$} & 1,3 & {$[1,7]$} \\
\hline Taxa de rotatividade $\left(\%\right.$ ano $\left.^{-1}\right)$ & 1,7 & 2,4 & {$[2,8]$} & 1,3 & {$[1,6]$} \\
\hline Taxa de mudança $\left(\%\right.$ ano $\left.^{-1}\right)(\%)$ & 0,4 & 0,8 & {$[1,1]$} & 0,2 & {$[0,4]$} \\
\hline
\end{tabular}

$10 \leq \mathrm{DAP}<20 \mathrm{~cm}$ a densidade aumentou na Borda e diminuiu no Interior. Entretanto, a distribuição de diâmetros em 2004 não diferiu significativamente da de 1999 para a amostra total $\left(\chi^{2}=6,480, P=0,166\right)$ bem como para a Borda $\left(\chi^{2}=4,328, P=0,228\right)$ e Interior $\left(\chi^{2}=3,154, P=0,532\right)$. Somente a análise das classes de DAP em separado indicou que o número de egressos (mortes mais emigrantes) superou significativamente o de ingressantes (recrutas + imigrantes) na classe de $5<$ DAP $<10 \mathrm{~cm}$ tanto para a amostra total como para ambos os estratos (tabela 2). Na classe de $20 \leq \mathrm{DAP}<40 \mathrm{~cm}$, o número de ingressantes superou significativamente o de egressos apenas na Borda. Não houve diferenças significativas nas demais classes de diâmetro. Houve diferença significativa na mortalidade entre as classes diamétricas para a amostra total $\left(\chi^{2}=11,923, P=0,018\right)$ e Interior $\left(\chi^{2}=12,732\right.$,
$P=0,013)$, mas não para a Borda $\left(\chi^{2}=2,881\right.$, $P=0,410)$. Nos dois primeiros casos, a mortalidade foi maior que a esperada na menor classe de diâmetro e menor que a esperada nas demais classes. Desta maneira, o processo geral de declínio da densidade e aumento da área basal se caracteriza principalmente pela redução da densidade de árvores da menor classe de diâmetro e crescimento de árvores sobreviventes, sobretudo nas classes de diâmetro maiores. $\mathrm{O}$ aumento da densidade de árvores maiores só foi claro na Borda. Dinâmica das populações arbóreas - Do total de 185 espécies arbóreas registradas pelos inventários de 1999 e 2004, cinco espécies só o foram no primeiro e dez no segundo, o que representa um acréscimo líquido, não significativo, de cinco espécies $(Z=1,29, P>0,05)$. Entre as 26 espécies mais abundantes, quatro não apresentaram mudanças líquidas no número de 


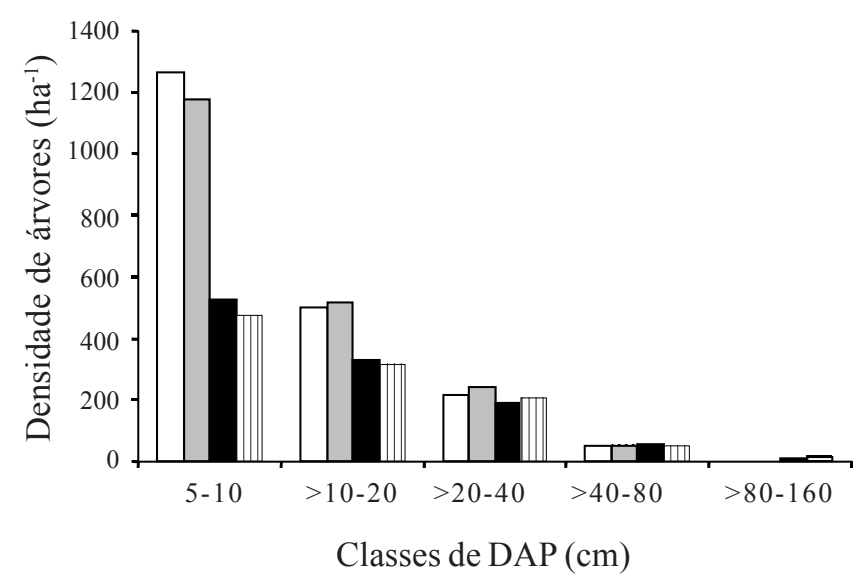

Figura 1. Distribuição por classe de diâmetro (DAP) da densidade de indivíduos arbóreos inventariados em 1999 e 2004 em um fragmento a floresta semidecídua em Piedade do Rio Grande, MG, nos estratos amostrais Borda e Interior. ( $\square=$ Borda 1999; $\square=$ Borda 2004; $\square=$ Interior 1999; $\otimes=$ Interior 2004).

Figure 1. Diameter $(\mathrm{DAP}=\mathrm{dbh})$ class distribution of the density of individual trees surveyed in 1999 and 2004 in a fragment of tropical semideciduous forest in Piedade do Rio Grande, SE Brazil, in the two sample strata, Edge (Borda) and Interior.

( $\square=$ Edge 1999; $\square$ = Edge 2004; $\square=$ = Interior 1999; 曲= Interior 2004).

indivíduos, mas tiveram aumento líquido de área basal; oito apresentaram aumento no número indivíduos e na área basal; sete apresentaram declínio no número indivíduos e na área basal; e somente seis reproduziram o padrão predominante na comunidade, isto é, declínio do número de indivíduos e aumento da área basal (tabela 3, figura 2).

Das cinco espécies que apresentaram declínio significativo do número de indivíduos, três tem habitat preferencial na Borda, uma no Interior e uma é indiferente ao habitat. Além disso, todas as três espécies que apresentaram aumento significativo do número de indivíduos tiveram habitat preferencial na Borda (tabela 3), o que caracteriza populações mais instáveis quanto à densidade entre as preferenciais de Borda e mais estáveis entre as preferenciais de Interior. A única exceção entre estas últimas foi Guapira opposita cuja densidade caiu significativamente. Das cinco espécies que apresentaram declínio significativo do número de indivíduos, uma é pioneira, três são de dossel-exigentes de luz e uma é de sub-dossel. As três com aumento significativo incluem uma pioneira, uma de dosselexigente de luz e uma de sub-dossel, não caracterizando nenhuma tendência quanto às guildas de regeneração. Todas as 16 espécies que registraram proporções significativamente diferentes de parcelas com área basal crescente ou decrescente também acusaram predominância da mudança crescente, em consonância com a tendência geral da comunidade (tabela 3 ). Os habitats preferenciais e guildas de regeneração, no entanto, não mostraram nenhuma tendência clara de distribuição entre as 16 espécies com área basal crescente ou as dez sem alteração significativa.

$\mathrm{O}$ aumento das taxas de rotatividade de número de indivíduos entre as 26 espécies corresponde a um aumento significativo das taxas de rotatividade de área basal ( $r=0,692, F=17,35, P=0,00009)$ e o mesmo se verifica entre as taxas de mudança de número de indivíduos e de área basal $(r=0,648, F=22,11$, $P=0,00035$ ) (figura 2). Espécies preferenciais de ambos os habitats e espécies indiferentes são observadas entre os valores mais baixos das taxas de rotatividade e mudança, mas espécies de Borda predominam entre os valores mais elevados. As exceções são Cestrum schlechtendalii e Prunus myrtifolia, espécies preferenciais de Interior, mas com alta rotatividade de número de árvores e área basal, respectivamente. Espécies de quase todas as guildas de regeneração se distribuem por toda a amplitude das taxas de mudança e rotatividade, exceto as espécies de dossel tolerantes à sombra, todas com baixa rotatividade de número de árvores e de área basal.

As comparações das taxas de mortalidade e recrutamento de cinco populações entre Borda e Interior (tabela 4) acusou diferença significativa apenas para a mortalidade de Metrodorea stipularis, maior no Interior que na Borda, e recrutamento de Mollinedia widgrenii, maior na Borda que no Interior. Nos demais casos, mortalidade e recrutamento não diferiram entre Borda e Interior.

\section{Discussão}

Em florestas tropicais maduras e não perturbadas, o padrão esperado para dinâmica de comunidades arbóreas no longo prazo é a estabilidade por meio do balanço entre as taxas de mortalidade e recrutamento e entre taxas de perda e ganho de biomassa em pé (Lang \& Knight 1983, Lieberman et al. 1985, Swaine et al. 1987, Rankin-de-Merona et al. 1990, Felfili 1995a, Santos et al. 1998). Além disso, tais florestas geralmente mostram uma mortalidade independente do tamanho para as árvores com diâmetros acima de 5 a $10 \mathrm{~cm}$ (Lieberman et al. 1985, Swaine et al. 1987, Swaine 1990). Os resultados do presente estudo, no entanto, rejeitaram a primeira hipótese, que previa a estabilidade 
Tabela 2. Dinâmica da comunidade arbórea por classe de diâmetro (DAP) entre 1999 e 2004 em um fragmento de floresta semidecídua em Piedade do Rio Grande, MG, contabilizada para a amostra total e seus dois estratos, Borda e Interior. As freqüências esperadas (esp.) para o número árvores em 2004 e para o número de mortos por classe de DAP basearam-se na distribuição do número de árvores por classe de DAP em 1999. As taxas são mortalidade anual; os valores seguintes são emigrantes (Emigr.), recrutas (Recr.), imigrantes (Imigr.) e comparações de Poisson entre as contagens de saídas (mortos+emigrantes) e entradas (recrutas+imigrantes).

Table 2. Tree community dynamics per diameter $(\mathrm{DAP}=\mathrm{dbh})$ classes between 1999 and 2004 in a fragment of tropical semideciduous forest in Piedade do Rio Grande, SE Brazil, given for the total sample and its two strata, Edge and Interior. Expected frequencies (esp.) for the number of trees in 2004 and the number of death events per DAP class were based on the distribution of the number of trees per DAP class in 1999. Rates are annual mortality; following values are outgrowth (Emigr.), recruits (Recr.), ingrowth (Imigr.), and Poisson count comparisons between outputs (death+outgrowth) and inputs (recruitment+ingrowth).

\begin{tabular}{|c|c|c|c|c|c|c|c|c|c|c|c|}
\hline \multirow{2}{*}{$\begin{array}{l}\text { DAP } \\
(\mathrm{cm})\end{array}$} & \multicolumn{3}{|c|}{$\mathrm{N}^{\circ}$ de árvores } & \multicolumn{3}{|c|}{ Mortos } & \multirow{2}{*}{$\begin{array}{c}\text { Emigr. } \\
\mathrm{N}^{\circ}\end{array}$} & \multirow{2}{*}{$\frac{\text { Recr. }}{\mathrm{N}^{\circ}}$} & \multirow{2}{*}{$\begin{array}{l}\text { Imigr. } \\
\mathrm{N}^{\circ}\end{array}$} & \multicolumn{2}{|c|}{ Cont. Poisson } \\
\hline & 1999 & 2004 & esp. & $\mathrm{N}^{\circ}$ & esp. & $\%$ ano $^{-1}$ & & & & $Z$ & $p$ \\
\hline \multicolumn{12}{|l|}{ Borda: } \\
\hline $5-10$ & 607 & 565 & $(586,5)$ & 82 & $(77,7)$ & 2,9 & 66 & 102 & 4 & 2,635 & 0,010 \\
\hline$>10-20$ & 241 & 249 & $(232,9)$ & 33 & $(30,9)$ & 2,9 & 27 & 1 & 67 & 0,707 & $\mathrm{~ns}$ \\
\hline$>20-40$ & 104 & 116 & $(100,5)$ & 8 & $(13,3)$ & 1,6 & 4 & - & 24 & 2,000 & 0,050 \\
\hline$>40-80$ & 24 & 24 & $(23,2)$ & 2 & $(3,1)$ & 1,7 & 2 & - & 3 & 0,378 & $\mathrm{~ns}$ \\
\hline$>80-160$ & - & - & & - & & & - & - & - & & \\
\hline Totais & 976 & 954 & & 125 & & 2,7 & 99 & 103 & 98 & & \\
\hline \multicolumn{12}{|l|}{ Interior: } \\
\hline $5-10$ & 379 & 344 & $(361,1)$ & 55 & $(39,1)$ & 3,1 & 26 & 45 & 1 & 3,106 & 0,002 \\
\hline$>10-20$ & 238 & 227 & $(226,8)$ & 17 & $(24,6)$ & 1,5 & 23 & - & 29 & 1,324 & $\mathrm{~ns}$ \\
\hline$>20-40$ & 138 & 148 & $(131,5)$ & 9 & $(14,2)$ & 1,3 & 4 & - & 22 & 1,521 & $\mathrm{~ns}$ \\
\hline$>40-80$ & 40 & 37 & $(38,1)$ & 2 & $(4,1)$ & 1,0 & 1 & - & 2 & 0,447 & $\mathrm{~ns}$ \\
\hline$>80-160$ & 9 & 10 & $(8,6)$ & - & $(0,9)$ & - & - & - & 1 & 1,000 & $\mathrm{~ns}$ \\
\hline Totais & 804 & 766 & & 83 & & 2,2 & 54 & 45 & 55 & & \\
\hline \multicolumn{12}{|c|}{ Amostra total: } \\
\hline $5-10$ & 986 & 909 & $(952,8)$ & 137 & $(115,2)$ & 2,9 & 92 & 147 & 5 & 3,945 & 0,001 \\
\hline$>10-20$ & 479 & 476 & $(462,9)$ & 50 & $(56,0)$ & 2,2 & 50 & 1 & 96 & 0,214 & $\mathrm{~ns}$ \\
\hline$>20-40$ & 242 & 264 & $(233,8)$ & 17 & $(28,3)$ & 1,4 & 8 & - & 47 & 2,593 & 0,010 \\
\hline$>40-80$ & 64 & 61 & $(61,8)$ & 4 & $(7,5)$ & 1,3 & 3 & - & 4 & 0,905 & $\mathrm{~ns}$ \\
\hline$>80-160$ & 9 & 10 & $(8,7)$ & - & $(1,1)$ & - & - & - & 1 & 1,000 & $\mathrm{~ns}$ \\
\hline Totais & 1780 & 1720 & & 208 & & 2,5 & 153 & 148 & 153 & & \\
\hline
\end{tabular}

da comunidade e populações arbóreas. Na verdade, os padrões de dinâmica no período do estudo revelaram um quadro de instabilidade, com mudanças claras caracterizadas pelo acúmulo de biomassa em pé (expresso pela área basal crescente), declínio da densidade de árvores, principalmente das de menor porte, e uma significativa mortalidade dependente do tamanho. Este quadro, conhecido como auto-desbaste, é freqüentemente associado a fases de construção intermediárias a tardias do ciclo silvigenético, isto é, fases avançadas de regeneração pós-distúrbio (Gentry \& Terborgh 1990, Oliveira Filho et al. 1997, Werneck \&
Franceschinelli 2004). De fato, distúrbios de diversos tipos são comumente a principal causa de instabilidade em florestas tropicais, o que inclui secas extraordinárias, tempestades, fogo, deslizamentos de terra, inundações e intervenções humanas (Condit et al. 1995, 1996, Laurance et al. 1998a, Whitmore \& Burslem 1998, Burslem \& Whitmore 1999, Laurance et al. 2001, Gomes et al. 2003, Sheil \& Burslem 2003, DamascenoJunior et al. 2004). As duas últimas fontes de distúrbios já foram, inclusive, associadas a processos de autodesbaste na mesma região (Oliveira Filho et al. 1997, Guilheme et al. 2004, Appolinário et al. 2005). No 


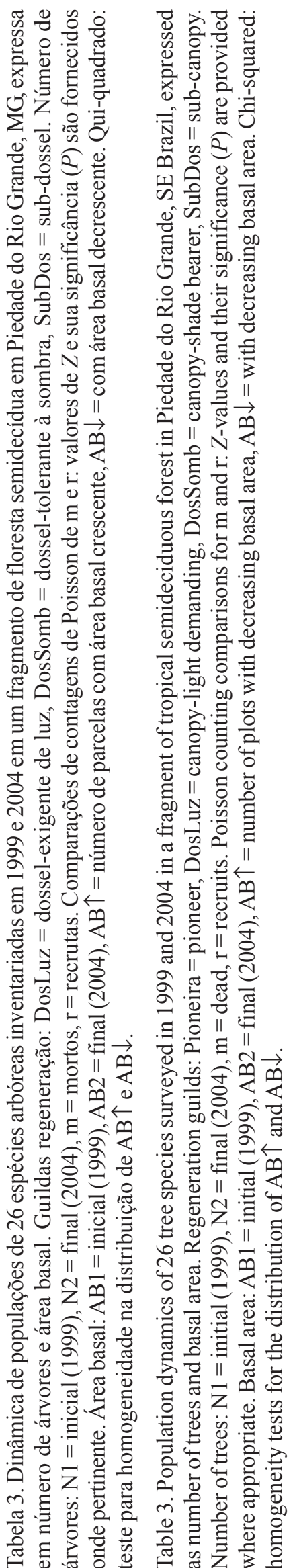

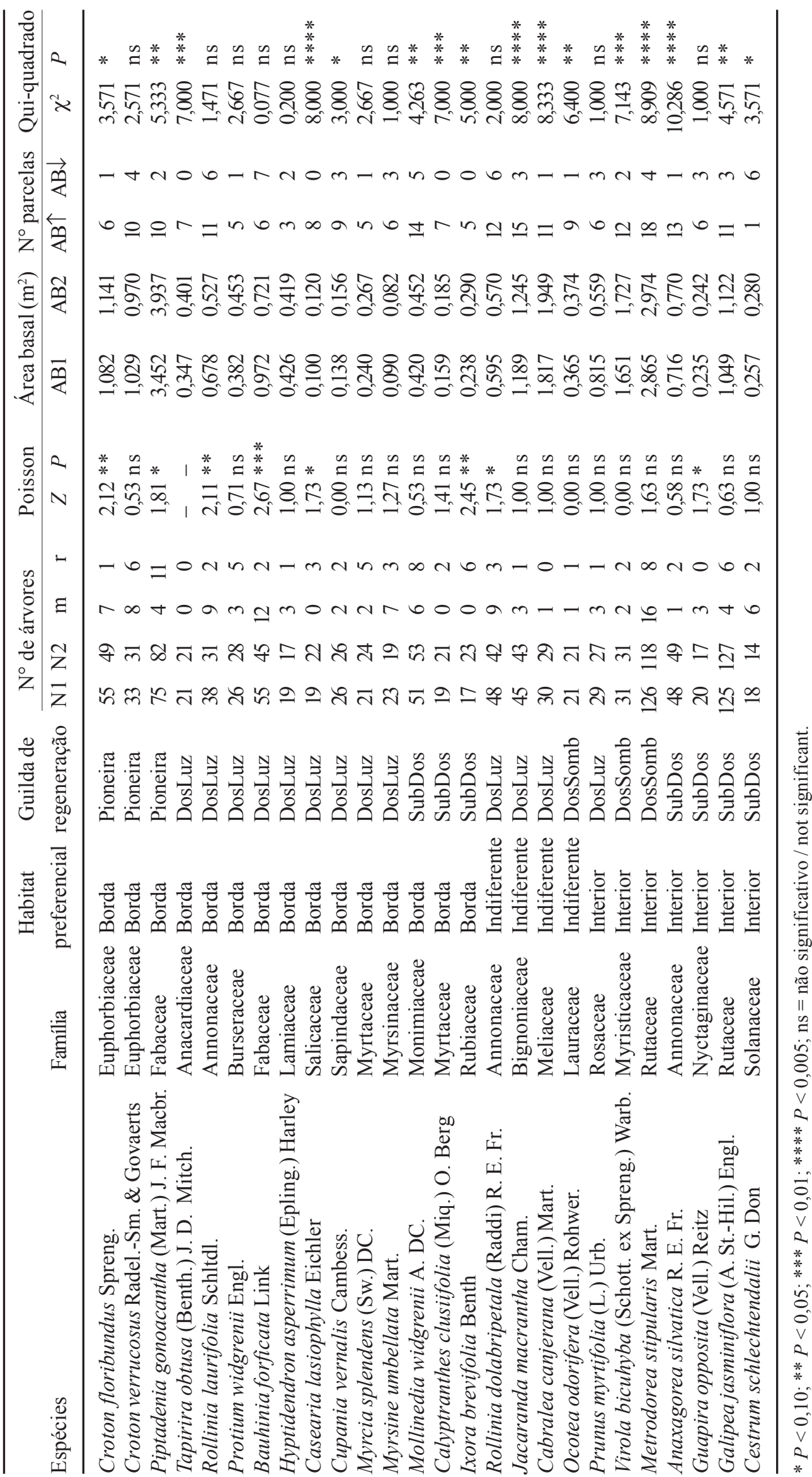



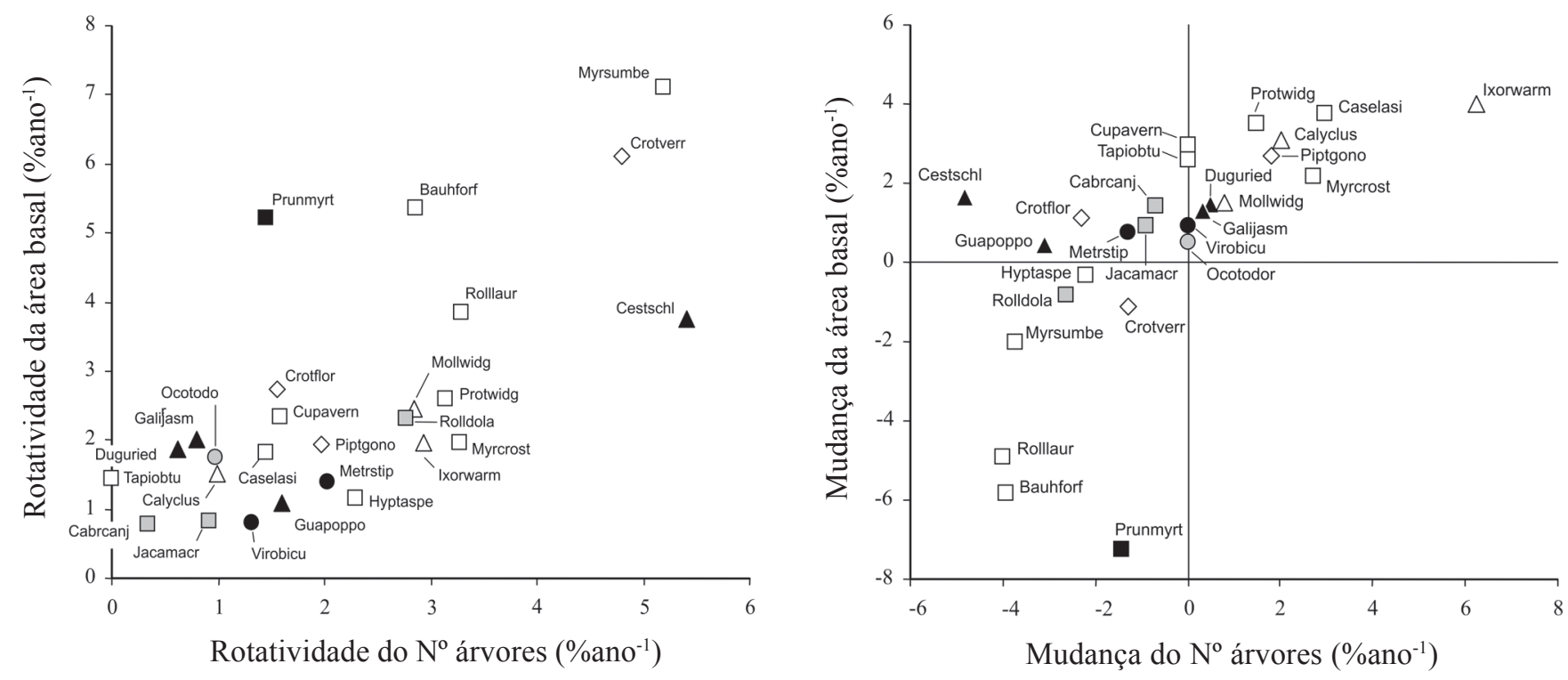

Figura 2. Dinâmica de populações de 26 espécies arbóreas inventariadas em 1999 e 2004 em um fragmento de floresta semidecídua em Piedade do Rio Grande, MG, expressa em rotatividade do número de árvores e área basal e mudança líquida do número de árvores e área basal. $\mathrm{O}$ nome das espécies está abreviado (nomes completos na tabela 3 ).

(Guildas de regeneração: $\diamond=$ Borda, Pioneira; $\square=$ Borda, dossel-exigente de luz; $\triangle=$ Borda, sub-dossel; $\square=$ Indiferente, dossel-exigente de luz; $\boldsymbol{\square}=$ Interior, dossel-exigente de luz; $\bullet=$ Interior, dossel-tolerante à sombra; $\mathbf{\Delta}=$ Interior, sub-dossel; - = Indiferente, dossel-tolerante à sombra).

Figure 2. Population dynamics of 26 tree species surveyed in 1999 and 2004 in a fragment of tropical semideciduous forest in Piedade do Rio Grande, SE Brazil, expressed as turnover of the number of trees and basal area and net change of the number of trees and basal area. Species' names are abbreviated (full names in table 3).

(Regeneration guilds: $\diamond=$ Edge, Pioneer; $\square=$ Edge, canopy-light demanding; $\triangle$ = Edge, sub-canopy; $\square=$ Indiferent, canopylight demanding; - = Interior, canopy-light demanding; $\bullet=$ Interior, canopy-shade bearer; $\boldsymbol{\Delta}=$ Interior, sub-canopy; - = Indiferent, canopy-shade bearer).

presente caso, entretanto, o processo geral de autodesbaste da comunidade não pôde ser vinculado a distúrbios generalizados sofridos pelo fragmento no período do estudo ou imediatamente anterior a ele. $\mathrm{O}$ distúrbio da fragmentação em si, mesmo que ocorrido há mais de 150 anos, deve ser a primeira causa a ser investigada. Sabe-se que florestas úmidas tropicais que sofreram cortes rasos restauram a densidade e a área basal originais em um período que varia de 50 a 150 anos (e.g. Uhl et al. 1982, Saldarriaga et al. 1988, Tabarelli \& Mantovani 1999, Saldarriaga \& Uhl 1991), o que indica tanto uma grande heterogeneidade como a possibilidade de efeitos de muito longo prazo. Apesar de o corte raso ser um distúrbio bem mais dramático que a fragmentação e da idade do fragmento estar no limite máximo sugerido pelos autores, a fragmentação não pode ser de todo descartada como uma causa remota do auto-desbaste detectado no período.

Distúrbios à parte, alguns autores também têm sugerido que períodos de instabilidade fazem parte dos ciclos rítmicos de algumas florestas tropicais não perturbadas, que alcançam um balanço por meio de períodos com maior mortalidade ou perda de biomassa, alternados com períodos de maior recrutamento ou acúmulo de biomassa, os quais podem estar associados a flutuações climáticas (Manokaran e Kochummen 1987, Swaine 1990, Phillips \& Gentry 1994, Felfili 1995a, Sheil et al. 2000). Devido à proteção estrita da área por parte dos proprietários nos últimos 150 anos e ao curto prazo de cinco anos do presente estudo, a hipótese dos ciclos dinâmicos, associados ou não a flutuações climáticas, também não pode ser descartada na explicação da fase de instabilidade registrada entre 1999 e 2004. Uma evidência a favor desta hipótese é o fato de não ter sido registrado no presente caso um declínio de espécies pioneiras e exigentes de luz e expansão de espécies tolerantes à sombra, típicos de processos de auto-desbaste na regeneração pós-distúrbio (Oliveira Filho et al. 1997, Sheil et al. 2000, Gomes et al. 2003, Werneck \& Franceschinelli 2004). Contudo, novos estudos se fazem necessários para verificar esta possibilidade. Uma outra hipótese, polêmica, mas 
Tabela 4. Dinâmica de populações de cinco espécies arbóreas inventariadas em 1999 e 2004 em um fragmento de floresta semidecídua em Piedade do Rio Grande, MG, expressa em número de árvores nos estratos amostrais da Borda e Interior. As autoridades das espécies são encontradas em Carvalho et al. (dados não publicados). Número de árvores: N1 = inicial (1999), $\mathrm{N} 2=$ final (2004), $\mathrm{m}=$ mortos, $\mathrm{r}=$ recrutas, $\mathrm{s}=$ sobrevivente. Teste $\chi^{2}$ para homogeneidade das razões m:s e r:s na Borda e Interior.

Table 4. Population dynamics of five tree species surveyed in 1999 and 2004 in a fragment of tropical semideciduous forest in Piedade do Rio Grande, SE Brazil, expressed as number of trees in the two sample strata: Edge (Borda) and Interior. Species' authorities are given in Carvalho et al. (unpublished data). Number of trees: $\mathrm{N} 1=$ initial (1999), N2 = final (2004), $\mathrm{m}=\mathrm{dead}$, $\mathrm{r}=$ recruits, $\mathrm{s}=$ ssurvivor. Chi-squared test for homogeneity of m:s and r:s ratios in the Edge and Interior.

\begin{tabular}{|c|c|c|c|c|c|c|c|c|c|c|c|c|c|c|}
\hline \multirow[b]{2}{*}{ Espécie } & \multicolumn{5}{|c|}{ Borda } & \multicolumn{5}{|c|}{ Interior } & \multicolumn{2}{|c|}{ Teste $\chi^{2} \mathrm{~m}: \mathrm{s}$} & \multicolumn{2}{|c|}{ Teste $\chi^{2} \mathrm{r}: \mathrm{s}$} \\
\hline & N1 & $\mathrm{N} 2$ & $\mathrm{~m}$ & $\mathrm{~s}$ & $\mathrm{r}$ & $\mathrm{N} 1$ & $\mathrm{~N} 2$ & $\mathrm{~m}$ & $\mathrm{~s}$ & $\mathrm{r}$ & $\chi^{2}$ & $p$ & $\chi^{2}$ & $\bar{p}$ \\
\hline Rollinia dolabripetala & 32 & 25 & 8 & 24 & 1 & 16 & 17 & 1 & 15 & 2 & 2,46 & ns & 0,92 & $\mathrm{~ns}$ \\
\hline Jacaranda macrantha & 21 & 21 & 1 & 20 & 1 & 24 & 22 & 2 & 22 & 0 & 0,23 & $\mathrm{~ns}$ & 1,073 & $\mathrm{~ns}$ \\
\hline Mollinedia widgrenii & 33 & 36 & 5 & 28 & 8 & 18 & 17 & 1 & 17 & 0 & 1,03 & $\mathrm{~ns}$ & 4,449 & $* *$ \\
\hline Galipea jasminiflora & 22 & 21 & 1 & 21 & 0 & 103 & 106 & 3 & 100 & 6 & 0,16 & $\mathrm{~ns}$ & 1,248 & $\mathrm{~ns}$ \\
\hline Metrodorea stipularis & 30 & 32 & 1 & 29 & 3 & 96 & 86 & 15 & 81 & 5 & 3,12 & $*$ & 0,468 & $\mathrm{~ns}$ \\
\hline
\end{tabular}

$* P<0,10 ; * * P<0,025 ;$ ns $=$ não significativo $/$ not significant.

plausível, é que o padrão encontrado no presente trabalho se encaixa na presumida aceleração global da rotatividade florestal nos últimos anos como resultado do incremento do $\mathrm{CO}_{2}$ atmosférico (Phillips \& Gentry 1994, Condit 1997, Phillips \& Sheil 1997). Outros estudos semelhantes que estão sendo conduzidos na região poderão verificar se o padrão se repete de forma generalizada em outras florestas maduras, reforçando esta hipótese.

A despeito da instabilidade detectada para a comunidade como um todo e para a maioria das populações analisadas, também foram verificados dois padrões de heterogeneidade espacial dos processos dinâmicos. $\mathrm{O}$ primeiro deles se refere às parcelas que registraram declínio da área basal e/ou aumento da densidade de árvores, diferentemente do processo predominante de auto-desbaste. $\mathrm{Na}$ verdade, tais parcelas certamente amostraram peças minoritárias do mosaico florestal que correspondem a fases de degradação (abertura de clareiras) e construção inicial do ciclo silvigenético, o que foi confirmado pelo registro da morte e queda de grandes árvores nestas parcelas entre os dois inventários. A queda de grandes árvores é um dos mais fortes fatores moduladores processo de dinâmica florestal no curto prazo, ocasionando perdas imediatas e consideráveis de biomassa em pé, que podem também ser acompanhadas de redução da densidade de árvores (Sheil et al. 2000). Tais distúrbios localizados desencadeiam as fases iniciais de sucessão, geralmente caracterizadas pela expansão tanto da biomassa como da densidade de árvores (Oliveira Filho et al. 1997, Gomes et al. 2003).
O segundo padrão de heterogeneidade espacial dos processos dinâmicos detectado refere-se à dicotomia entre a borda e o interior do fragmento florestal, confirmando a segunda hipótese do trabalho, que preconizava uma dinâmica mais acelerada na borda que no interior. De fato, apesar de as taxas de dinâmica do número de indivíduos e da área basal serem mais aceleradas na borda que no interior, o balanço entre elas resultou em padrões de instabilidade com balanço semelhante nos dois ambientes. Diversos estudos sobre fragmentação florestal nos trópicos têm demonstrado que as bordas apresentam condições microclimáticas distintas das do interior da floresta ou da floresta contínua, que incluem maior luminosidade e temperatura, maior exposição aos ventos, e menor umidade atmosférica e do solo (Kapos 1989, Kapos et al. 1997, Bierregaard Jr. et al. 2001). Essas diferenças afetam a dinâmica da comunidade vegetal e a mortalidade e recrutamento diferenciais levam, com o tempo, à instalação de comunidades com composição de espécies distinta da original e, portanto, da comunidade do interior dos fragmentos (Múrcia 1995, Viana et al. 1997, Metzger 1999). No presente caso, Carvalho et al. (dados não publicados) já haviam reconhecido a diferenciação da composição da flora arbórea em relação à do interior, como resultado da antiguidade das bordas (ca. 150 anos). Estudos realizados na Amazônia têm demonstrado que a fragmentação de florestas tropicais leva ao incremento das taxas de mortalidade e recrutamento imediatamente após a formação das bordas como resultado da alteração das condições microclimáticas (Laurance et al. 1998a, 1998b, 2001, 
Bierregaard Jr. et al. 2001). Mesmo no caso de bordas mais antigas ou mesmo bordas naturais nas transições floresta-savana a dinâmica das comunidades arbóreas de borda se distingue das de interior, normalmente com taxas de rotatividade mais altas (Felfili 1995a, 1995b, Oliveira Filho et al. 1997, Viana et al. 1997, van den Berg \& Santos 2002).

A maior luminosidade e exposição aos ventos tem sido associada à maior rotatividade nas bordas, mas deve-se salientar que, depois de certo tempo, aumenta a proporção de espécies pioneiras e exigentes de luz que normalmente têm crescimento mais rápido e ciclo de vida mais curto que espécies tolerantes à sombra (Lieberman \& Lieberman 1987), o que contribui ainda mais para o incremento da rotatividade nas bordas. De fato, os maiores valores de rotatividade, tanto de número de árvores como área basal, foram registrados entre espécies pioneiras e exigentes de luz preferenciais de borda, ao passo que os menores valores se concentraram entre as espécies de dossel tolerantes à sombra, preferenciais de interior ou indiferentes ao habitat. As comparações da dinâmica de número de indivíduos entre sub-populações de borda e interior detectou um número muito pequeno de diferenças significativas, sugerindo que as diferenças na dinâmica da comunidade entre os dois ambientes se deve mais à composição diferenciada de espécies que à dinâmica diferenciada entre subpopulações.

As populações de espécies preferenciais de borda, além de registrarem os maiores valores de rotatividade, também mostraram um maior número de casos de instabilidade quanto à densidade que as espécies de interior e indiferentes aos dois habitats. Esta maior instabilidade pode se vincular ao fato de que tais espécies são em geral pioneiras ou exigentes de luz de ciclo mais curto, com maior investimento em reprodução e crescimento rápido que em habilidade competitiva (Swaine \& Whitmore 1988), o que pode resultar em oscilações de suas abundâncias relativas em resposta a flutuações ambientais. De fato, entre as populações instáveis da borda, três declinaram e três se expandiram no período, sugerindo um balanço entre elas.

Os padrões de diferenciação espacial da dinâmica da comunidade e populações arbóreas do fragmento estudado refletem uma considerável heterogeneidade ambiental que testemunha a riqueza e complexidade da cobertura florestal contínua primitiva na região. A fragmentação florestal trouxe a intromissão do efeito borda, que incrementou fortemente a heterogeneidade ambiental pré-existente e vinculada às variações do substrato e ao mosaico sucessional da floresta. Este fato salienta a importância do tamanho dos fragmentos florestais para promover a conservação de amostras das comunidades, tamanho este que dever ser grande o suficiente para acomodar os processos diferenciados das bordas e preservar os processos primitivos no interior dos fragmentos.

Agradecimentos - Este trabalho contou com apoio financeiro da Fundação de Amparo à Pesquisa de Minas Gerais (FAPEMIG) e do Programa de Pós-graduação em Engenharia Florestal da Universidade Federal de Lavras. Agradecemos ainda o apoio logístico e a carinhosa hospedagem dos anfitriões Galdino e Sueli Carvalho.

\section{Referências bibliográficas}

APPOLINÁRIO, V., OLIVEIRAFILHO, A.T. \& GUILHERME F.A.G. 2005. Tree population and community dynamics in a Brazilian tropical semideciduous forest. Revista Brasileira de Botânica 28:347-360.

BIERREGAARD JR., R.O., LOVEJOY, T.E., KAPOS, V., SANTOS, A.A. \& HUTCHINGS, R.W. 1992. The biological dynamics of tropical rainforest fragments. Bioscience 42:859-866.

BIERREGAARD JR., R.O., GASCON, C., LOVEJOY, T.E. \& MESQUITA, R.C.G. 2001. Lessons from Amazonia: the ecology and conservation of a fragmented forest. Yale University Press, London.

BURSLEM, D.F.R.P. \& WHITMORE. T. 1999. Species diversity, susceptibility to disturbance and tree population dynamics in tropical rain forests. Journal of Vegetation Science 10:767-776.

BRASIL. 1992. Normais climatológicas (1961-1990). Ministério da Agricultura, Departamento Nacional de Meteorologia, Brasília.

BRASIL. 2002. Biodiversidade brasileira - Avaliação e identificação de áreas e ações prioritárias para conservação, utilização sustentável e repartição dos benefícios da biodiversidade nos biomas brasileiros. Brasília, Ministério do Meio Ambiente, Secretaria de Biodiversidade e Florestas.

CONDIT, R. 1997. Forest turnover, diversity, and CO2. Trends in Ecology and Evolution 12:249-250.

CONDIT, R., HUBBELL, S.P. \& FOSTER, R.B. 1995. Mortality rates of 205 Neotropical tree and shrub species and the impact of a severe drought. Ecological Monographs 65:419-439.

CONDIT, R., HUBBEL, S.P. \& FOSTER, R.B. 1996. Changes in tree species abundance in a Neotropical forest: impact of climate change. Journal of Tropical Ecology 12:231-256.

DAMASCENO JUNIOR, G.A., SEMIR, J., SANTOS, F.A.M. \& LEITÃO FILHO, H.F. 2004. Tree mortality in a riparian forest at Rio Paraguai, Pantanal, Brazil, after an extreme flooding. Acta Botanica Brasilica 18:839-846. 
FELFILI, J.M. 1995a. Growth, recruitment and mortality in the Gama gallery forest in central Brazil over a six-year period (1985-1991). Journal of Tropical Ecology 11:67-83.

FELFILI, J.M. 1995b. Diversity, structure and dynamics of a gallery forest in central Brazil. Vegetatio 117:1-15

FUNDAÇÃO SOS MATA ATLÂNTICA. 2002. Atlas da evolução dos remanescentes florestais e ecossistemas associados da Mata Atlântica no período 1995-2000. http://www.sosmatatlantica.org.br (acesso em 22/03/2005).

GENTRY, A.H. \& TERBORGH, J. 1990. Composition and dynamics of the Cosha Cashu 'mature' floodplain forest. In Four Neotropical rainforests (A.H. Gentry, ed.). Yale University Press, New Haven, p.542-564.

GOMES, E.P.C., MANTOVANI, W. \& KAGEYAMA, P.Y. 2003. Mortality and recruitment of trees in a secondary montane rainforest in southeastern Brazil. Brazilian Journal of Biology 63:47-60.

GUILHERME, F.A.G., OLIVEIRAFILHO,A.T.,APPOLINÁRIO, V. \& BEARZOTI, E. 2004. Effects of flooding regimes and woody bamboos on tree community dynamics in a section of tropical semideciduous forest in SouthEastern Brazil. Plant Ecology 174:19-36.

KAPOS, V. 1989. Effects of isolation on the water status of forest patches in the Brazilian Amazon. Journal of Tropical Ecology 5:173-185.

KAPOS, V., CAMARGOS, J.L.C. \& DANDE, G. 1997. Edge related changes in environment and plant response due to forest fragmentation in Central Amazonia. In Tropical forest remnants: ecology, management and conservation of fragmented communities (W.F. Laurance \& R.O. Bierregaard, eds.). University of Chicago Press, Chicago, p.45-54.

KORNING, J. \& BALSLEV, H. 1994. Growth and mortality of trees in Amazonian tropical rain forest in Ecuador. Journal of Vegetation Science 4:77-86.

LANG, G.E. \& KNIGHT, D.H. 1983. Tree growth, mortality, recruitment, and canopy gap formation during a 10year period in a tropical moist forest. Ecology 64:1075-1080.

LAURANCE, W.F. 1997. Hyper-disturbed parks: edge effects and the ecology of isolated rainforest reserves in tropical Australia. In Tropical forest remnants: ecology, management and conservation of fragmented communities (W.F. Laurance \& R.O. Bierregaard, eds.). University of Chicago Press, Chicago, p.71-83.

LAURANCE, W.F. \& YENSEN, E. 1991. Predicting the impacts of edge effects in fragmented habitats. Biological Conservation 55:77-92.

LAURANCE, W.F., FERREIRA, L.V., RANKIN-DE-MERONA, J.M. \& LAURANCE, S.G. 1998a. Rain forest fragmentation and the dynamics of Amazonian tree communities. Ecology 79:2032-2040.
LAURANCE, W.F., FERREIRA, L.V., RANKIN-DE-MERONA, J.M., LAURANCE, S.G., HUTCHINGS, R.W.\& LOVEJOY, T.E. 1998b. Effects of forest fragmentation on recruitment patterns in Amazonian tree communities. Conservation Biology 12:460-464.

LAURANCE, W.F., WILLIAMSON, G.B., DELAMÔNICA, P., OLIVEIRA, A., LOVEJOY, T.E., GASCON, C. \& POHL, L. 2001. Amazonian wet forest resistance to the $1997-98 \mathrm{El}$ Niño drought. Conservation Biology 14:1538-1542.

LIEBERMAN, D. \& LIEBERMAN, M. 1987. Forest tree growth and dynamics at La Selva, Costa Rica (1969-1982). Journal of Tropical Ecology 3:347-358.

LIEBERMAN, D., LIEBERMAN, M., PERALTA, R. \& HARTSHORN, G.S. 1985. Mortality patterns and stand turnover rates in a wet tropical forest in Costa Rica. Journal of Ecology 73:915-924.

LOVEJOY, T.E., BIERREGAARD, R.O., RYLANDS, A.B., MALCON, J.R., QUINTELA, C.E., HARPER, L.H., BROWN, K.S., POWELL, A.H., POWELL, G.V.N., SCHUBART, H.O.R. \& HAYS, M.B. 1986. Edge and other effects of isolation on Amazon forest fragments. In Conservation biology: the science of scarcity and diversity. (M.E. Soulé, ed.). Sinauer Press, Massachusetts, p.257-285.

MANOKARAN, N. \& KOCHUMMEN, K.M. 1987. Recruitment, growth and mortality of the species in lowland dipterocarp forest in Peninsular Maylasia. Journal of Tropical Ecology 3:315-330.

METZGER, J.P. 1999. Estrutura da paisagem e fragmentação: análise bibliográfica. Anais da Academia Brasileira de Ciências 71:445-463.

MORRISON, D.F. 1976. Multivariate Statistical Methods, McGraw-Hill, New York.

MURCIA, C. 1995. Edges effects in fragmented forest: Implications for conservation. Trends in Ecology and Evolution 10:58-62.

NASON, J.D., ALDRICH, P.R. \& HAMRICK, J.L. 1997. Dispersal and the dynamics of genetic structure in fragmented tropical tree populations. In Tropical forest remnants: ecology, management and conservation of fragmented communities (W.F. Laurance \& R.O. Bierregaard Jr., eds.). University of Chicago Press, Chicago, p.304-320.

OLIVEIRAFILHO,A.T. \& FONTES M,A.L. 2000. Patterns of floristic differentiation among Atlantic forests in southeastern Brazil, and the influence of climate. Biotropica 32:793-810.

OLIVEIRAFILHO,A.T., VILELA, E.A., GAVILANES, M.L.\& CARVALHO, D.A. 1994. Comparison of the woody flora and soils of six areas of montane semideciduous forest in southern Minas Gerais, Brazil. Edinburgh Journal of Botany 51:355-389.

OLIVEIRAFILHO, A.T., MELLO, J.M. \& SCOLFORO, J.R.S. 1997. Effects of past disturbance and edges on tree community structure and dynamics within a fragment of tropical semideciduous forest in south-eastern Brazil over a five year period (1987-1992). Plant Ecology 131:45-66. 
PHILLIPS, O.L. \& GENTRY, A.H. 1994. Increasing turnover through time in tropical forests. Science 263:954-958.

PHILLIPS, O.L. \& SHEIL D. 1997. Forest turnover, diversity and $\mathrm{CO}_{2}$. Tree 12:404.

RANKIN-DE-MERONA, J.M., HUTCHINGS, H.R.W. \& LOVEJOY, T.E. 1990. Tree mortality and recruitment over a five-year period in undisturbed upland rainforest of the Central Amazon. In Four Neotropical rainforests (A.H. Gentry, ed.). Yale University Press, New Haven, p.573-584.

SALDARRIAGA, J.G. \& UHL, C. 1991. Recovery of forest vegetation following slash-and-burn agriculture in the upper Rio Negro. In Tropical rain forests: regeneration and management (A. Gómez-Pompa, T.C. Whitmore \& M. Hadley, eds.). Blackwell, New York, p.303-312.

SALDARRIAGA, J.G., WEST, D.C. \& THARP, M.L. 1988. Long-term chronosequence in the upper Rio Negro of Colombia and Venezuela. Journal of Ecology 76:938-958.

SANTOS, F.A.M., PEDRONI, F., ALVES, L.F. \& SANCHEZ, M. 1998. Structure and dynamics of tree species of the Atlantic Forest. Anais da Academia Brasileira de Ciências, 70:874-880.

SHEIL, D. \& BURSLEM, D.F.R.P. 2003. Disturbing hypotheses in tropical forests. Trends in Ecology and Evolution 18:18-26.

SHEIL, D., BURSLEM, D.F.R.P. \& ALDER, D. 1995. The interpretation and misinterpretation of mortality rate measures. Journal of Ecology 83:331-333.

SHEIL, D., JENNINGS, S. \& SAVILL, P. 2000. Long-term permanent plot observations of vegetation dynamics in Budongo, a Ugandan rain forest. Journal of Tropical Ecology 16:765-800.

SWAINE, M.D. 1990. Population dynamics of tree species in tropical forests. In Tropical forests: botanical dynamics, speciation and diversity (L.B. Holm-Nielsen, I.C. Nielsen \& H. Balslev, eds.). Academic Press, London, p.101-110.

SWAINE, M.D. \& WHITMORE, T.C. 1988. On the definition of ecological species groups in tropical rain forests. Vegetatio 75:81-86.
SWAINE, M.D., LIEBERMAN, D. \& PUTZ, F.E. 1987. The dynamics of tree populations in tropical forest: a review. Journal of Tropical Ecology 3:359-366.

TABARELLI, M. \& MANTOVANI, W. 1999. A regeneração de uma floresta tropical montana após corte e queima (São Paulo, Brasil). Revista Brasileira de Biologia 59:239-250.

TURNER, I.M. 1996. Species loss in fragments of tropical rainforests: a review of the evidence. Journal of Applied Ecology 33:200-209.

UHL, C., JORDAN, C., CLARK, K., CLARK, H. \& HERRERA, R. 1982. Ecosystem recovery in Amazon caatinga forest after cutting, cutting and burning, and bulldozer clearing treatments. Oikos 38:313-320.

VAN DEN BERG, E. \& SANTOS, F.A.M. 2002. Population dynamics of tree species of a riparian forest in southeastern Brazil. In 45th Symposium of the International Association for Vegetation Science (V.P. Pillar, coord.). IAVS, Porto Alegre, p.162.

VELOSO, H.P., RANGEL FILHO, A.L.R. \& LIMA, J.C. 1991. Classificação da vegetação brasileira adaptada a um sistema universal. IBGE, Rio de Janeiro.

VIANA, V.M., TABANEZ, A.J.A. \& BATISTA, J.L.F. 1997. Dynamics and restoration of forest fragments in the Brazilian Atlantic moist forest. In Tropical forest remnants: ecology, management and conservation of fragmented communities (W.F. Laurance \& R.O. Bierregaard Jr., eds.). The University of Chicago Press, London, p.351-365.

WERNECK, M. \& FRANCESCHINELLI, E.V. 2004. Dynamics of a dry forest fragment after the exclusion of human disturbance in southeastern Brazil. Plant Ecology 174:337-346.

WHITMORE, T.C. \& BURSLEM, D.F.R.P. 1998. Major disturbances in tropical rainforests. In Dynamics of tropical communities (D.M. Newbery, H.H.T. Prins \& N. Brown, eds.). Blackwell, Oxford, p.549-565.

YOUNG, A., BOYLE, T. \& BROWN, A. 1996. The population genetic consequences of habitat fragmentation. Trends in Ecology and Evolution 11:413-418.

ZAR, J.H. 1996. Biostatistical analysis, $3^{\text {rd }}$ ed. Prentice-Hall, New Jersey. 